\title{
Maynooth Optical Aeronomical Facility
}

\author{
Francis J. Mulligan \\ St. Patrick's College \\ Department of Experimental Physics \\ Maynooth, Co. Kildare, Ireland \\ E-mail: fmulligan@vax1.may.ie \\ Rick J. Niciejewski \\ University of Michigan \\ Space Physics Research Laboratory \\ Department of Atmospheric, Oceanic and \\ Space Sciences \\ 2455 Hayward \\ Ann Arbor, Michigan 48109
}

\begin{abstract}
Ground-based measurements of upper atmospheric parameters, such as temperature and wind velocity, can be made by observing airglow emissions that have a well-defined altitude profile and that are known to be representative of the emitting region. We describe the optical observatory at Maynooth $\left(53.23^{\circ} \mathrm{N}, 6.4^{\circ} \mathrm{W}\right)$ at which two instruments, a Fabry-Pérot interferometer and a Fourier transform spectrometer, are used to record atmospheric airglow emissions in Ireland at visible and near-infrared wavelengths, respectively. Descriptions of the instruments, data acquisition, and analysis procedures are provided, together with some sample results.
\end{abstract}

Subject terms: optical observatories; airglow observations; ground-based; mesospheric measurements.

Optical Engineering 33(12), 3853-3860 (December 1994).

\section{Introduction}

Measurements of atmospheric parameters such as temperature and wind velocity, as a function of time and location, are important in helping to elucidate the complex dynamical and chemical processes that influence atmospheric composition. Balloon ${ }^{1}$ and rocket-borne ${ }^{2}$ experiments have enabled atmospheric scientists to make measurements in the troposphere and stratosphere, while satellites such as Dynamics Explorer II have provided global coverage at much higher altitudes. ${ }^{3}$ The mesopause, located near an altitude of $87 \mathrm{~km}$, has proved to be one of the most difficult regions to study experimentally, being too high for balloon and too low for satellite observations. The majority of observations in this region have been obtained either by rocket sounding or by remote sensing of naturally occurring emissions in the infrared, visible, or ultraviolet spectrum. Rocket experiments provide excellent coverage in altitude at a particular time and location, but they are not capable of providing long-term measurements. Lidar techniques offer the greatest potential of providing high-resolution temperature and wind measurements at stratospheric and mesospheric altitudes, but it is only very recently that lidar techniques have developed so that the region near the mesopause can be studied using this technique. ${ }^{4}$ The measurement of atmospheric temperature and wind velocity by observing airglow emissions confined to narrow altitude bands continues to be one of the most costeffective methods available.

Essentially all airglow studies are made during the hours of darkness, because of the difficulty of discriminating against the overwhelming solar background during daylight hours. Many measurements have been made at high latitudes, where good weather conditions, together with continuous darkness in winter, allow long series of observations to be obtained. ${ }^{5-7}$ Ireland is not an ideal location in which to place an observing

Paper OI-024 received Apr. 25, 1994; revised manuscript received Aug. 29, 1994 accepted for publication Aug. 29, 1994.

(C) 1994 Society of Photo-Optical Instrumentation Engineers. 0091-3286/94/\$6.00 site for ground-based optical observations. Poor and very variable weather conditions together with high relative humidity and the absence of suitable high-altitude observing sites pose the greatest problems. The very difficulty of obtaining such measurements, however, renders their significance all the greater, particularly in helping to constrain global atmospheric models that often suffer from a lack of data points in certain latitude and longitude sectors. Figure 1 taken from Ref. 8 shows the global distribution of optical stations involved in upper atmosphere studies. ${ }^{8}$ Ireland's position on the western perimeter of Europe represents an important location in filling the void between the United States and mainland Europe.

Quite distinct from the role that an atmospheric observing site in Ireland can play as an element in a global grid of stations, its latitude is in the center of the range where high altitude $(\sim 82 \mathrm{~km})$ noctilucent clouds (NLCs) are observed during twilight in summer. NLCs are observed most frequently in the latitude region $50^{\circ}$ to $60^{\circ}$ on either side of the equator in summer months, when the sun is between $6^{\circ}$ and $16^{\circ}$ below the horizon. They are important in mesospheric physics because they lie close to the mesopause, and because it appears that their frequency of occurrence is on the increase. It is believed that this may be related to increasing concentrations of methane coupled with decreasing mesospheric temperatures. The ideal zone for viewing them ${ }^{9}$ is $53^{\circ}$ to $57^{\circ}$. From this point of view, Ireland should be a good location from which to undertake a study of the correlation between noctilucent cloud formation and mesopause temperature.

The role of internal gravity waves in maintaining the thermal structure of the mesopause is currently the subject of a number of investigations. ${ }^{10-12}$ These waves propagate through the stratosphere and mesosphere and dissipate their energy near the mesopause. Tropospheric convection, orographic effects, and even large-scale ocean waves are believed to be sources of these waves. Ireland's location at the edge of a vast expanse of open water with a prevailing westerly wind suggests that it might also be a good place from 


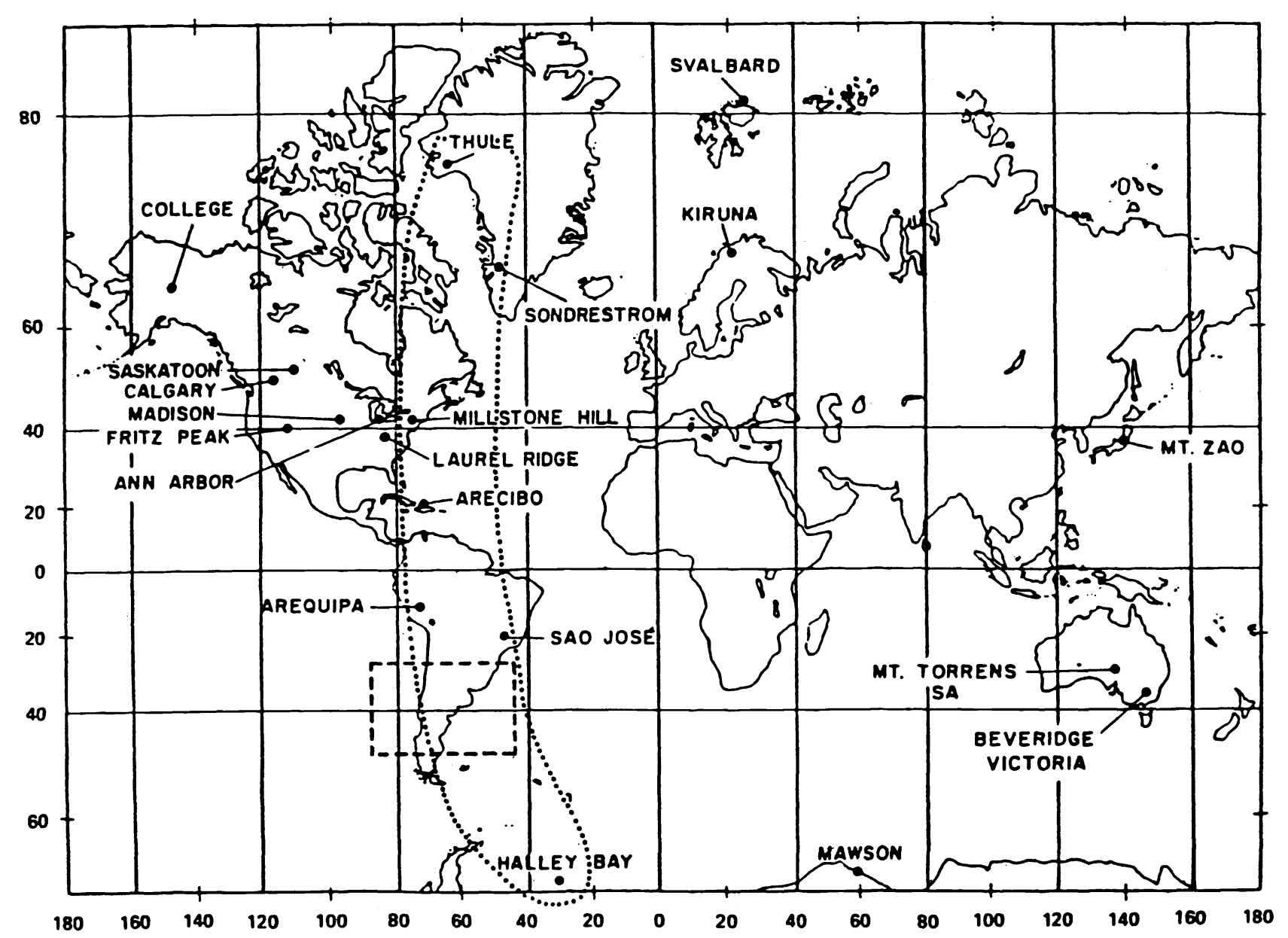

Fig. 1 Worldwide map of optical atmospheric observatories (after Ref. 8).

which to search for short-period (10 to $20 \mathrm{~min}$ ) gravity waves, since it has been suggested that the interaction of strong winds with mountains might be a source of lee-type gravity waves. ${ }^{12}$

This paper describes a small optical observatory located on the campus at Maynooth College $\left(53.2^{\circ} \mathrm{N}, 6.4^{\circ} \mathrm{W}\right)$, which houses a Fourier transform spectrometer and a Fabry-Pérot interferometer, for the purpose of measuring temperature and wind velocity in the upper mesosphere and lower thermosphere region. Descriptions of the instruments, data acquisition, and analysis procedures are provided, together with some sample results. Data recorded at Maynooth form part of a ground-based validation campaign for the Upper Atmosphere Research Satellite, through a collaboration with the Space Physics Research Laboratory of the University of Michigan.

\section{Instrument Descriptions}

\subsection{Fourier Transform Spectrometer}

The Fourier transform infrared (FTIR) spectrometer is based on a Michelson interferometer design and was supplied by Bomem Inc. A thermoelectrically cooled InGaAs detector is employed, which has maximum sensitivity in the wavelength region 1 to $1.65 \mu \mathrm{m}$. This range includes the rotational bands associated with $(\Delta v=2)$ vibrational transitions of the hydroxyl molecule. An internal He-Ne laser provides accurate data sampling, and the instrument has a maximum resolution of $2 \mathrm{~cm}^{-1}$. In this study, the resolution is set at $4 \mathrm{~cm}^{-1}$, which enables a complete interferogram to be acquired in about $5 \mathrm{~s}$ and represents a compromise between integration time and resolution. The instrument has a field of view of $\approx 1.5 \mathrm{deg}$ and typically 50 interferograms are coadded to increase the signal-to-noise ratio. The composite interferogram is apodized using the Hanning window, and a spectrum is obtained by Fourier transforming the apodized interferogram. The relative spectral response of the detector is determined by calibration with a low brightness source (LBS) whose area is sufficient to completely fill the field of view of the instrument and is similar in construction to that described by Dandekar and Davis. ${ }^{13}$ The calibration of the low brightness source is traceable to a primary standard, and we estimate that radiances calculated on the basis of this source are accurate to 10 to $15 \%$. Figure 2 , recorded shortly after midnight on 5 September 1993 with an integration time of $4.5 \mathrm{~min}$, is a typical spectrum showing both the $\mathrm{OH}(3,1)$ and $(4,2)$ vibration-rotation bands. The altitude of maximum emission of these bands in the night airglow is near $87 \mathrm{~km}$.

\subsection{Fabry-Pérot Interferometer}

The Fabry-Pérot interferometer (FPI) was designed and constructed in the Department of Experimental Physics at Maynooth College, with a view to recording the spectral line 


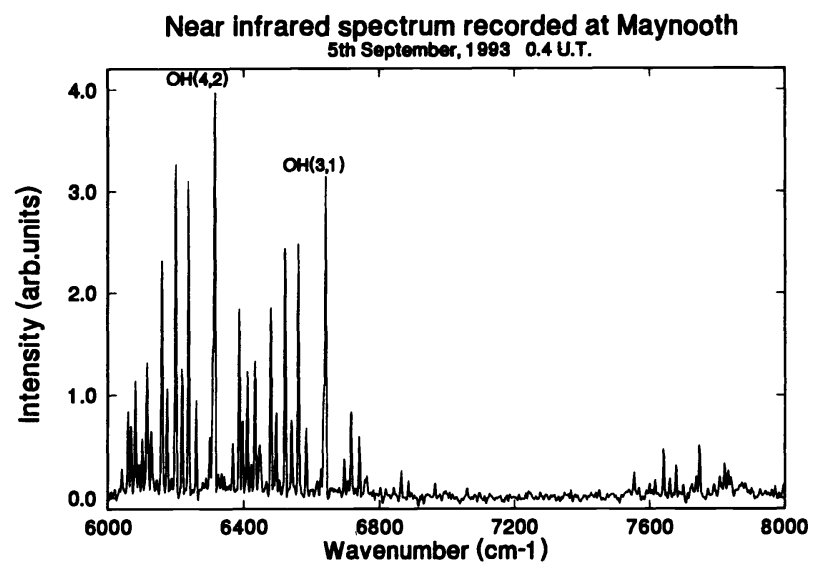

Fig. 2 Near-infrared spectrum recorded shortly after midnight at Maynooth on 5 September 1993 (integration time: $4.5 \mathrm{~min}$ ).

profile associated with the atomic oxygen emissions at 557.7 and $630 \mathrm{~nm}$; wavelength selection is achieved using narrow passband filters ( 2 and $6 \AA$, respectively). Details of the instrument parameters are listed in Table 1. The detector, which was provided by the University of Michigan, is one of a series of devices developed for the Fabry-Pérot interferometer on board the Dynamics Explorer II satellite ${ }^{14}$ and has also been used for ground-based studies at high latitudes. ${ }^{15}$ It comprises an S20 photocathode of diameter $1.2 \mathrm{~cm}$, three microchannel plates as the gain element, and an anode comprising 12 concentric annuli each of equal area. The device operates as if it were 12 distinct photomultiplier tubes, with a separate counting channel for each anode. Dark counts are reduced by cooling the detector using a thermoelectric cooler, which reduces the temperature at the cathode by about $40^{\circ} \mathrm{C}$ below ambient. Condensation on the detector window is prevented by the use of a thermopane window.

The Fabry-Pérot plates are optically contacted using three zerodur spacers, $1 \mathrm{~cm}$ in height, and are mounted in a rigid invar cage enclosed in a pressure-sealed aluminium housing. Fine parallelism adjustment is achieved by applying pressure to the plates over the zerodur spacers via invar springs. The contact area between the housing and the Fabry-Pérot plates is kept to a minimum to reduce the conduction of heat of the étalon through the mount.

Fabry-Pérot fringes are focused onto the detector via a $1-\mathrm{m}$ focal length lens. The optical system is such that the 12 channels of the detector intercept about $60 \%$ of a full free spectral range. The choice of optical parameters is a compromise between obtaining a good description of a line profile in the 12 channels, i.e., that the sky profile straddles at least 6 to 7 channels, while at the same time enabling the background signal level to be established accurately. Positioning of an interference fringe in the center of the 12 channels is achieved by changing the pressure in the sealed étalon housing via a motor-driven pressure controller, which is under the control of the data acquisition computer. The pressure controller also plays a vital role in determining the instrument response as described in Sec. 3.

The control and interface electronics are a combination of commercial units and laboratory specific modules designed and constructed with the measurements in mind. The high
Table 1 Maynooth Fabry-Pérot interferometer characteristics.

Plate diameter
Plate separation
Reflectivity (R) at $557.7 \mathrm{~nm}$
Free Spectral range
Instrumental finesse
Objective lens
Field of view
Interference filter
Detector

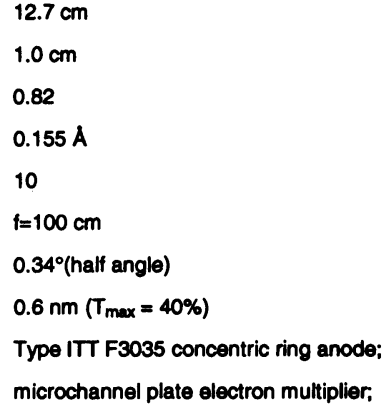

$12.7 \mathrm{~cm}$

$1.0 \mathrm{~cm}$

0.82

$0.155 \AA$

10

$f=100 \mathrm{~cm}$

$0.34^{\circ}$ (half angle)

$0.6 \mathrm{~nm}\left(T_{\max }=40 \%\right)$

Type ITT F3035 concentric ring anode;

microchannel plate electron multiplier;

S20 photocathode; $12 \mathrm{~mm}$ diameter (TE

cooled)

Spectral calibration

Signal integration time

Data acquisition and control

Frequency stabilized $\mathrm{He}-\mathrm{Ne}$ laser

5 minutes for intensities > $100 R$

80386 computer and CAMAC modules voltage for the detector together with the preamplifiers, discriminators, and pulse shapers were built at the University of Michigan. This dedicated electronics is controlled by analog and digital I/O lines from the data acquisition computer. Pulse counting is achieved through the use of CAMAC timer and counter modules, which are also under the control of the computer. A simplified block diagram of the entire instrument is shown in Fig. 3. Figure 4(a), recorded on 26 September 1993 at 2:34 universal time (U.T.) with an integration period of $2 \mathrm{~min}$, is a typical sky profile obtained when observing the atomic oxygen $\mathrm{O}^{1} \mathrm{~S} 557.7-\mathrm{nm}$ emission. At midlatitudes, this emission comes predominantly from an altitude near $95 \mathrm{~km}$. Channels 10 and 12 have considerably different dark

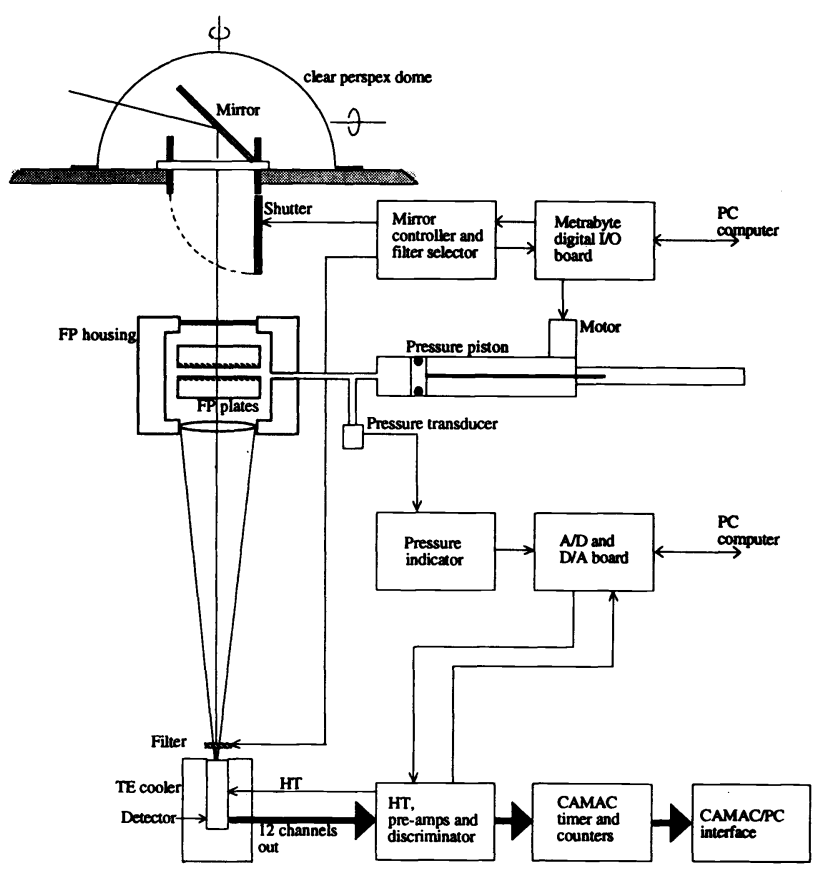

Fig. 3 Block diagram of Maynooth Fabry-Pérot interferometer. 


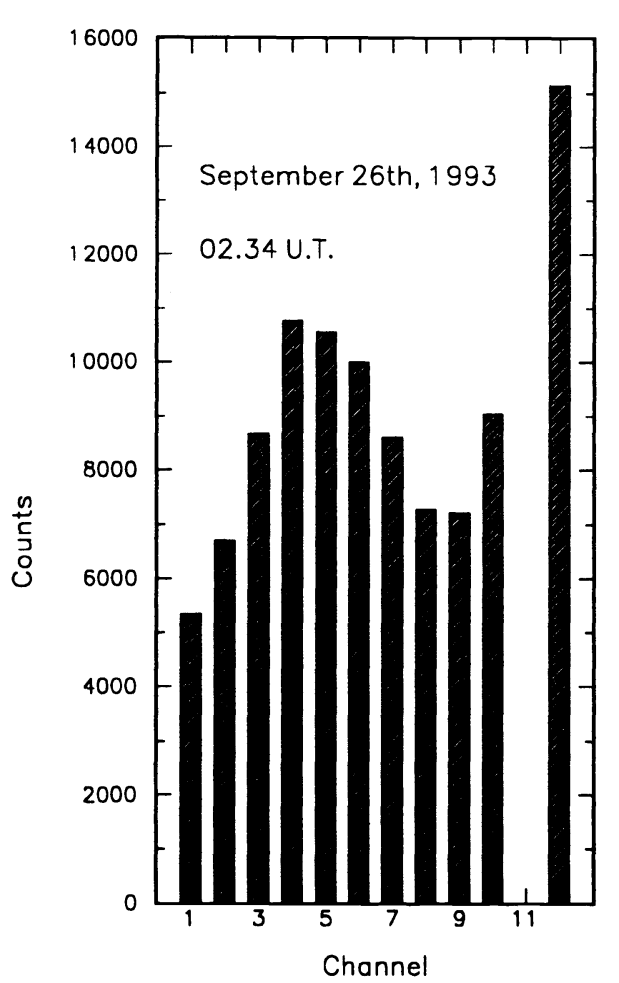

(a)

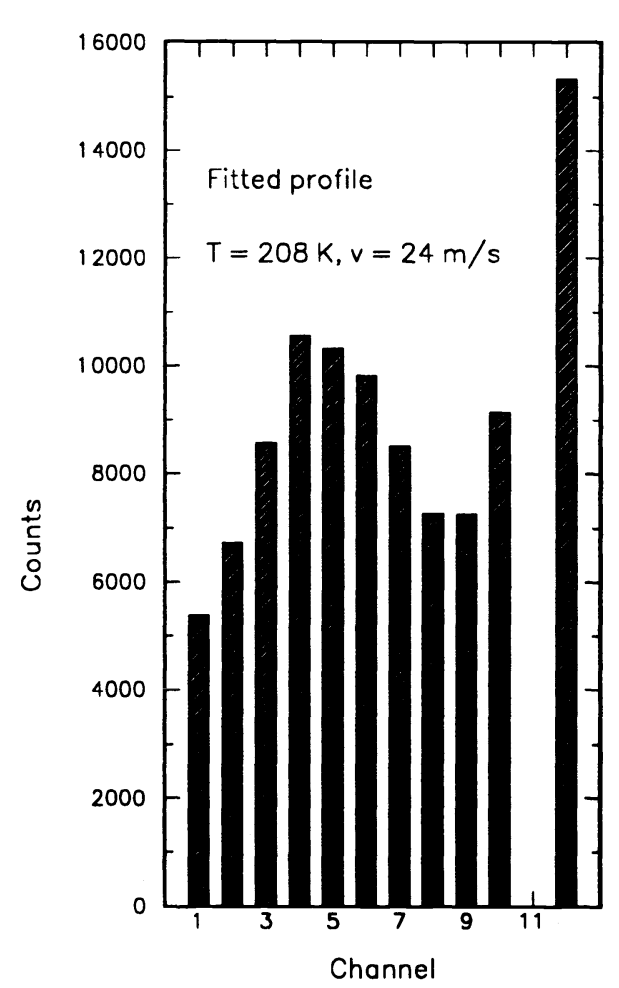

(b)

Fig. 4 (a) O'S 557.7-nm profile recorded at Maynooth on 26 September 1993 at 2.34 UT (integration time: $2 \mathrm{~min}$ ), (b) Best-fit synthetic $\mathrm{O}^{1} \mathrm{~S} 557.7-\mathrm{nm}$ profile (in least-squares sense) to that shown in (a) for an emission temperature of $208 \mathrm{~K}$ and a line-of-sight velocity of $24 \mathrm{~m} / \mathrm{s}$.

counts and sensitivity compared with channels 1 to 9 as indicated in Fig. 4(a). The absence of counts in channel 11 was caused by the fact that channel 11 was inoperative in the detector employed in this study.

\section{Temperature Calculation Technique}

\subsection{OH Emission}

Rotational temperatures for hydroxyl emissions are determined from the intensity distribution of the rotational lines. The lifetime of the vibrational state is sufficiently long-lived for the emitting molecule to reach thermal equilibrium with its surroundings ${ }^{16}$; the rotational levels are thus populated according to a Boltzmann distribution. For an emission at a temperature $T_{\text {rot }}$, the intensity of a rotational line is given by $^{17}$ :

$I_{\left(J^{\prime}, v^{\prime} \rightarrow J^{\prime \prime}, v^{\prime \prime}\right)}=$

$N_{v^{\prime}} A_{\left(J^{\prime}, \nu^{\prime} \rightarrow J^{\prime \prime}, \nu^{\prime \prime}\right)} \frac{2\left(2 J^{\prime}+1\right)}{Q_{\nu^{\prime}}\left(T_{\text {rot }}\right)} \exp \left[\frac{-E_{v^{\prime}}\left(J^{\prime}\right)}{k T_{\text {rot }}}\right]$,

where $N_{v^{\prime}}$ is the number of molecules in the $v^{\prime}$ vibrational level, $J^{\prime}$ is the upper rotational state angular momentum, $A_{\left(J^{\prime}, v^{\prime} \rightarrow J^{\prime \prime}, v^{\prime \prime}\right)}$ is the Einstein coefficient for spontaneous emission from vibration-rotation state $\left(J^{\prime}, v^{\prime}\right)$ to state $J^{\prime \prime}, \nu^{\prime \prime}$, and $E_{v^{\prime}}\left(J^{\prime}\right)$ is the term value of the upper state. The term $Q_{v^{\prime}}$ is the partition function or state sum and is given by
$Q_{\nu^{\prime}}\left(T_{\text {rot }}\right)=\sum_{J^{\prime}} 2\left(2 J^{\prime}+1\right) \exp \left[\frac{-E_{\nu^{\prime}}\left(J^{\prime}\right)}{k T_{\text {rot }}}\right]$

Equation (1) may be converted to a linear equation in the following form:

$\ln \left\{\frac{I}{2\left(2 J^{\prime}+1\right) A_{\left(J^{\prime}, \nu^{\prime} \rightarrow J^{\prime \prime}, \nu^{\prime \prime}\right)}}\right\}=\ln \left\{\frac{N_{\nu^{\prime}}}{Q_{\nu^{\prime}}\left(T_{\text {rot }}\right)}\right\}-\frac{E_{\nu^{\prime}}\left(J^{\prime}\right)}{k T_{\text {rot }}}$.

The rotational temperature can be obtained from the slope of a plot of the left-hand side versus $E_{v^{\prime}}\left(J^{\prime}\right)$. For an emitting source in thermal equilibrium, such a plot is a straight line. The uncertainty of the linear fit is a measure of the temperature uncertainty. The intercept on the plot may be used to determine the intensity of the emission.

Instrument response is measured at the beginning and end of each observing night. Following a night of observations, each spectrum of the form shown in Fig. 2 is corrected for instrument response and processed according to the method outlined above. In this study, the three most intense lines $P_{1}(2), P_{1}(3)$, and $P_{1}(4)$ of the transition from vibrational state 3 to vibrational state 1 are used to obtain the temperature. These are among the most intense lines in the spectrum and are not affected to any significant extent by water vapor absorption. ${ }^{17}$ This results in a sequence of temperature and intensity values for the night in question. Figure 5(a), which 


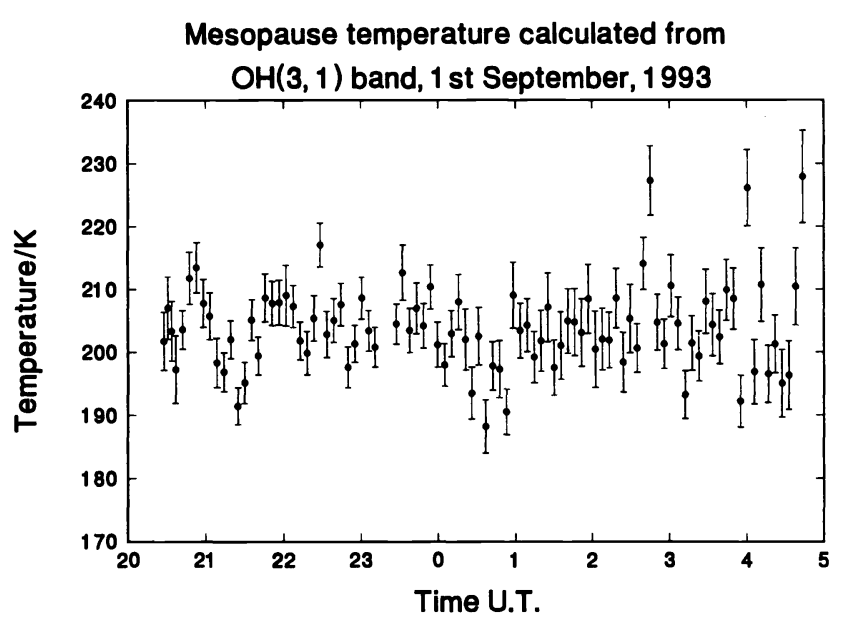

(a)

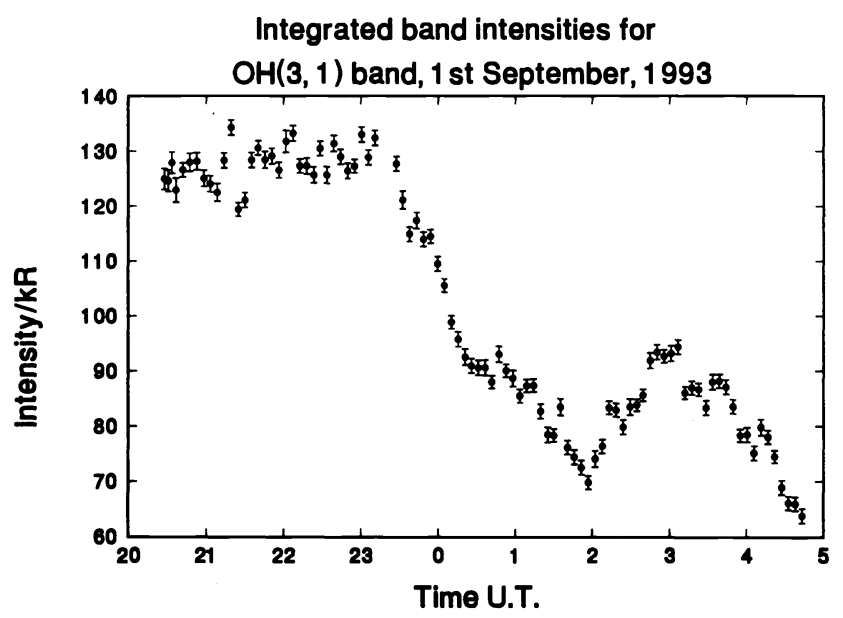

(b)

Fig. 5 (a) Temperature values calculated from $\mathrm{OH}(3,1)$ spectra recorded by the Maynooth FTIR on night of 1-2 September 1993. (b) Integrated band intensities of $\mathrm{OH}(3,1)$ emission recorded by the Maynooth FTIR on night of 1-2 September 1993.

shows the variation in derived temperature during the night of 1-2 September 1993, is typical of the results obtained throughout the year. Einstein coefficients were obtained from Mies. ${ }^{18}$ Partition function values were obtained from Krassovsky, Shefov, and Yarin, ${ }^{19}$ while rotational term values were taken from Coxon. ${ }^{20}$ Figure 5(b) shows the corresponding intensity variations for the same night. Statistical errors on both rotational temperature and band intensity obviously depend on airglow intensity, which was found to vary by as much as a factor of 3 during a single night. At times of strong $\mathrm{OH}$ emission, statistical uncertainty in the calculated rotational temperatures dropped to as low as $\pm 2 \mathrm{~K}$. Typically, about 170 spectra were recorded in a single night during midwinter, decreasing to only about 50 in midsummer.

\subsection{Oxygen $\mathrm{O}^{1} \mathrm{~S}$ Emission}

The Doppler broadening of an emission line, with an unshifted nominal wavelength $\lambda_{0}$ from a source in thermal equilibrium at a temperature $T$ is given as
$\Delta \lambda_{\mathrm{D}}=\frac{2 \lambda_{0}}{c}\left[\frac{2 k T \ln (2)}{m}\right]^{1 / 2}$,

where $k$ is Boltzmann's constant, $c$ is the speed of light, and $m$ is the mass of the emitting atom. Any line-of-sight Doppler shift $\delta \lambda$ is used to determine a wind velocity vector $v$ from the relation

$\nu=\frac{\delta \lambda}{\lambda_{0}} c$.

Combining observations from each of the cardinal directions (north, south, east, west) allows a wind velocity vector to be determined for the observing station.

The spectral line recorded by the Fabry-Pérot interferometer is a convolution of the Doppler broadened emission line source and instrumental broadening. It is therefore necessary to deconvolve these two effects before attributing a temperature to the emitting region. In this study, the instrumental profile was determined by recording the output from the instrument when illuminated uniformly by a frequency stabilized He-Ne laser that emits a spectral line of negligible Doppler width at $632.8 \mathrm{~nm}$. The response of each of the 12 detector channels is recorded as a function of pressure over three free spectral ranges. This allows the calculation of the instrumental response in terms of a series of Fourier sine and cosine coefficients for each channel as described by Killeen and Hays. ${ }^{21}$ These coefficients are then corrected for the change in wavelength from $632.8 \mathrm{~nm}$ to the wavelength of the oxygen $\mathrm{O}^{1} \mathrm{~S}$ emission, which is at $557.7 \mathrm{~nm}$. Synthetic profiles are generated by convolving the instrument description with generated Gaussian profiles for known values of temperature, wind velocity, signal, and background intensity. A nonlinear least-squares fitting approach is used to determine the optimum fit between the generated and recorded profile. The values of temperature, wind velocity, signal, and background intensity are then assigned to the emitting altitude at the time of the observation. Figure 4(b) shows the best-fit synthetic profile to the measured profile shown in Fig. 4(a) for an emission temperature of $208 \mathrm{~K}$, and a line-of-sight wind velocity of $24 \mathrm{~m} / \mathrm{s}$. Figure 6(a) shows an example set of temperatures recorded on 26-27 September 1993 using this method, while Fig. 6(b) shows the corresponding zonal wind velocities.

\section{Environmental Considerations and Data-Taking Procedure}

The observatory was designed to allow the two instruments to function in daily unattended operation. The observer typically initiates the observing program before leaving in the evening; the program then waits until the sky becomes suitably dark as determined by a light sensor, before commencing observations. With the approach of dawn the instruments cease observing and return to the dormant state. The layout of the observatory, with separate rooms for the Fabry-Pérot interferometer, Fourier transform spectrometer, and control room, is shown in Fig. 7.

The temperature of the room housing the Fabry-Pérot interferometer is controlled to better than $0.1^{\circ} \mathrm{C}$ so as to avoid unintended shifts in the position of the fringe peak due to temperature effects. Light from the sky enters the room through a clear Perspex dome and is directed toward the 


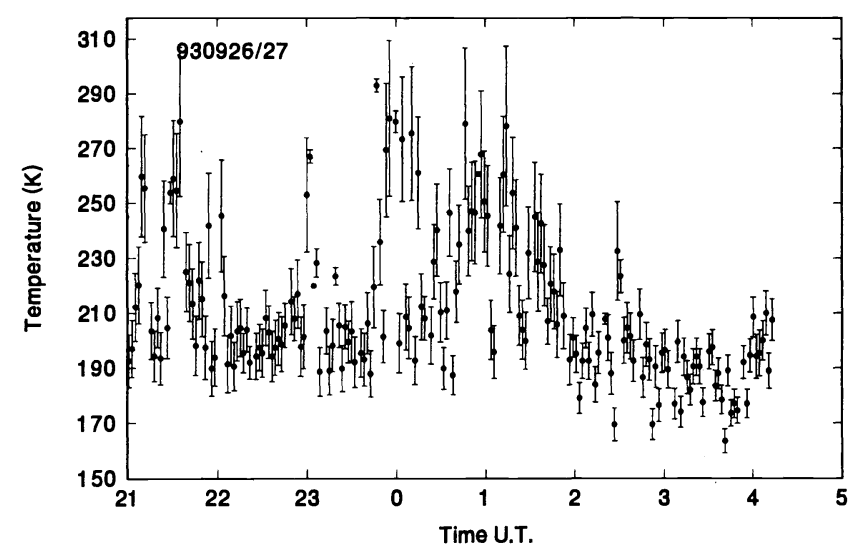

(a)

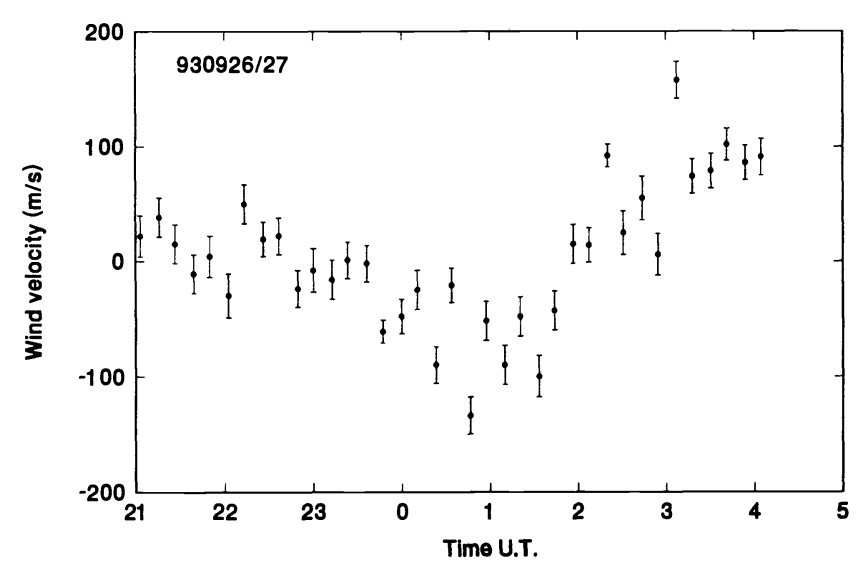

(b)

Fig. 6 (a) Temperature values calculated from O'S 557.7-nm profiles recorded by the Maynooth FPI on night of 26-27 September 1993. (b) Zonal wind velocities (east is positive) as a function of time from 557.7-nm emission recorded at Maynooth on night of 26-27 September 1993.

Fabry-Pérot interferometer by a mirror system mounted in the dome. The position of the mirror in azimuth and altitude is controlled by the data acquisition computer. A small ring heater positioned at the base of the dome dissipating about $250 \mathrm{~W}$ of heat is sufficient to keep the dome clear of condensation at all times. During calibrations, light from the sky is prevented from entering the room by a shutter, which acts as a reflection screen for the calibration source.

The FTIR instrument has been designed to be used in demanding conditions, and it does not have the stringent environmental requirements of the Fabry-Pérot interferometer. An identical instrument has been employed to obtain measurements from an aircraft during the Airborne Lidar and Observations of the Hawaiian Airglow Campaign (ALOHA-90). ${ }^{22}$ It is supplied with data acquisition software from the manufacturer, which can be tailored to the user's needs through the use of keystroke macros. This instrument was placed in a second room in the observatory that was fitted with an opening window, made from clear Perspex. We decided to record all sky data with the window in the open position, due to the presence of many absorption bands in Perspex at infrared wavelengths. At the start of observations for a particular night, the window is opened and a timer is

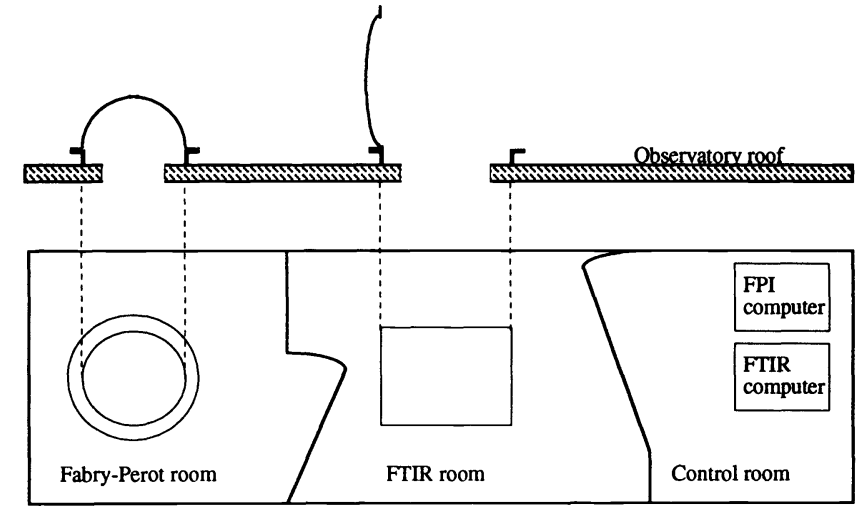

Fig. 7 Layout of observatory at Maynooth.

started. The instrument is protected from the elements by a rain detector, which if activated causes the window to close and the timer to stop. Unfortunately, any data recorded after the time of the window closing has to be discarded. Intensity calibration of the FTIR is performed by replacing the input mirror by a demountable low brightness source.

\section{Results}

\subsection{Annual Variations in Mesopause Temperature}

Figure 8 shows the times during which $\mathrm{OH}$ observations were obtained at Maynooth during 1993. The FTIR instrument was functional at all times during the year, and the gaps are caused by unsuitable weather conditions. This figure is included here as an indicator of the fraction of time available for observations in Ireland. An average value of temperature has been calculated for each night on which data were recorded at Maynooth during 1993 and is shown in Fig. 9. The well-known winter-summer variation for a midlatitude station, with the minimum in summer and maximum in winter, is clearly evident in these data. The generally accepted explanation of this apparent inversion is that the cold summer (warm winter) mesopause is maintained through adiabatic cooling (warming) produced by a strong summer-to-winter meridional cell that is driven by breaking vertically propagating gravity waves. ${ }^{23}$ Also shown in the figure are the temperature results obtained by Clancy and Rusch ${ }^{24}$ from data recorded by the Solar Mesosphere Explorer (SME) satellite for an altitude of $86.5 \mathrm{~km}$ at a latitude of $50^{\circ} \mathrm{N}$. The close correspondence between the SME results and the data recorded in this study helps to confirm that the instrument performance and methods used to calculate temperatures are reliable. Figure 9 also shows the predictions of the MSISE-90 model $^{25}$ for an altitude of $87 \mathrm{~km}$ at a latitude of $53^{\circ} \mathrm{N}$. The model predicts a mean temperature of $185.7 \mathrm{~K}$, which is some $15 \mathrm{~K}$ below that measured in this study. Results of a harmonic analysis applied to both the model and the measured temperatures are shown in Table 2 and are in very good agreement.

\subsection{Combined Results from Both Instruments}

It is widely believed that temperature fluctuations at mesopause altitudes, which have timescales of the order of minutes to hours, are the result of vertically propagating acoustic gravity waves from lower in the atmosphere..$^{6,7,17}$ Noxon ${ }^{26}$ was the first to suggest that conclusive evidence for the pas- 


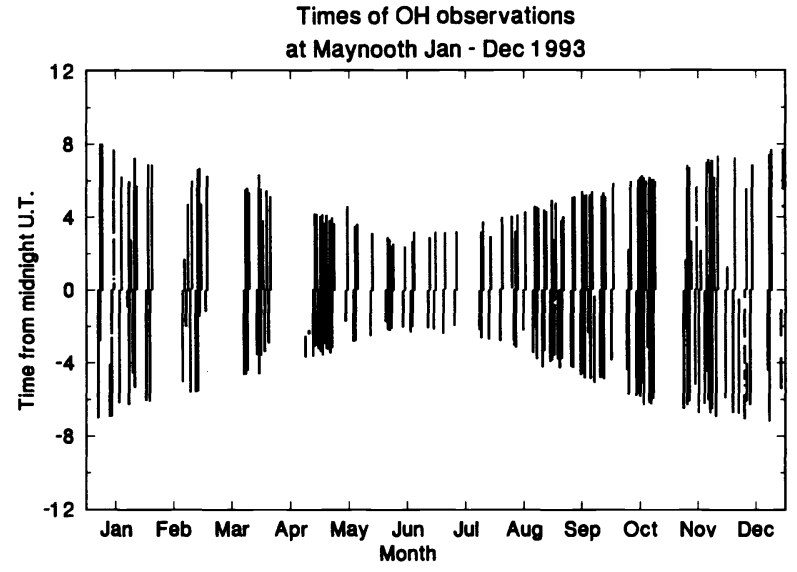

Fig. 8 Times at which $\mathrm{OH}$ spectra were recorded at Maynooth during 1993.

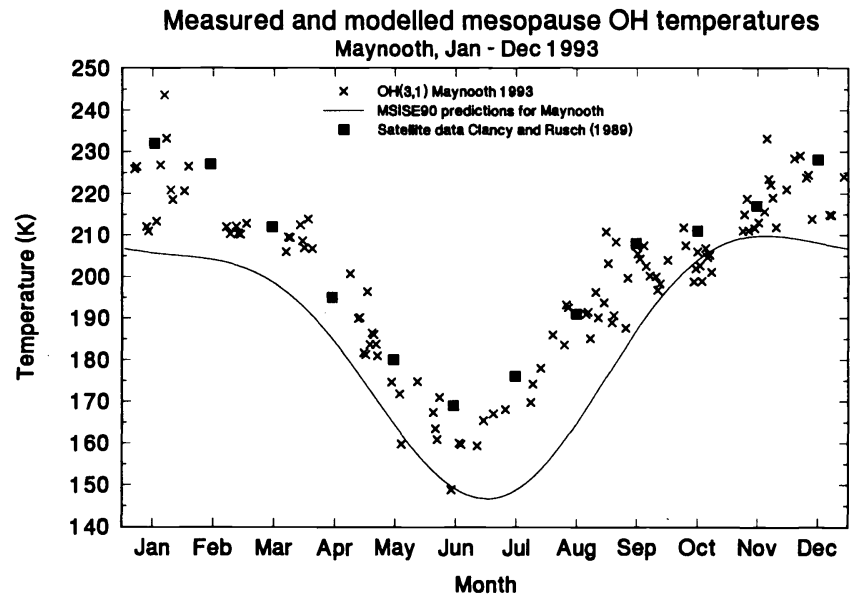

Fig. 9 Comparison of nightly averaged temperatures measured by the Maynooth FTIR with results from Clancy and Rusch ${ }^{24}$ and predictions of MSISE-90 for the Maynooth station.

sage of an atmospheric gravity wave could be obtained by observing simultaneously different airglow emissions originating from two different altitude regions. The altitude of the peak emission for the $\mathrm{OH} \mathrm{band} \mathrm{d}^{27}$ is centered near $87 \mathrm{~km}$, while the $\mathrm{O}^{1} \mathrm{~S}$ emission ${ }^{28}$ maximizes at $95 \mathrm{~km}$ in midlatitudes with a small contribution from the thermosphere near $250 \mathrm{~km}$. It must be recalled, of course, that these layers are several kilometers in depth and that these are the altitudes of the peak emissions only. Assuming that the emissions represent the altitudes at the peak of the emission, we can calculate parameters associated with vertically propagating acoustic gravity waves. Figure 10 shows temperature values calculated from both the $\mathrm{OH}$ emission and the $\mathrm{O}^{1} \mathrm{~S}$ atomic oxygen emission for the night of 27-28 September 1993. To emphasize the correlation between the two data sets, the atomic oxygen results, which are representative of the higher altitude, have been shifted backward in time by $20 \mathrm{~min}$. This is the result that one might expect, assuming that the temperature fluctuation observed in both emissions is the result of a wave motion propagating upward from below the mesopause. The phase lag between the two fluctuations has been used to estimate a vertical velocity, assuming that the altitude difference between the two layers is known. A knowledge of
Table 2 Observed $[\mathrm{OH}(3,1)$ results for 1993] and predicted (MSISE-90) mean, annual, and semiannual variations in mesopause temperature at Maynooth.

\begin{tabular}{|c|c|c|c|c|c|}
\hline \multicolumn{2}{|c|}{} & \multicolumn{2}{c|}{ Annual component } & \multicolumn{2}{c|}{ Semiannual component } \\
\hline & Mean temp./K & Amplitude/K & Phase/days & Amplitude(K) & Phase/days \\
\hline OH(3,1) & 200 & $27 \pm 1$ & $95 \pm 2$ & $7 \pm 1 \mathrm{~K}$ & $-51 \pm 9$ \\
MSISE-90 & 185.68 & $30.1 \pm 0.2$ & $95.3 \pm 0.6$ & $9 \pm 0.2 \mathrm{~K}$ & $-99 \pm 1$ \\
\hline
\end{tabular}

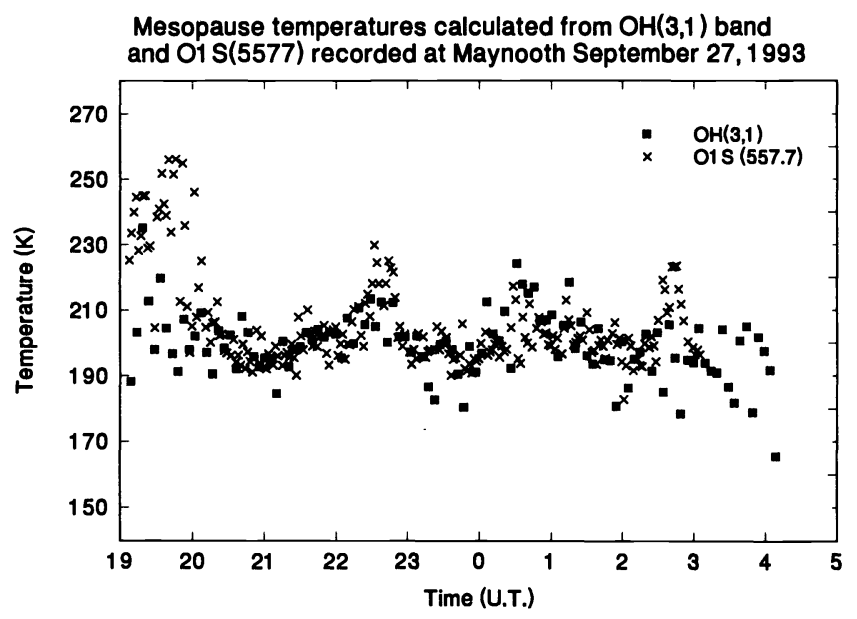

Fig. 10 Combined temperature results for both FPI and FTIR on night of 27-28 September 1993. The FPI results have been shifted backward in time by 20 min to illustrate the correspondence between the two separate data sets.

the vertical velocity and the wave period, which can be obtained from either data set, allows the vertical and horizontal wavelength to be determined. ${ }^{29}$ These are important parameters in developing models of gravity wave propagation through the mesosphere. ${ }^{30}$ Table 3 presents a list of the parameters that were calculated from the two data sets recorded at Maynooth on 27-28 September 1993. The values of period, vertical, and horizontal wavelength deduced are consistent with the summary of gravity wave parameters reported by Manson. ${ }^{31}$

\section{Summary}

The Fourier transform spectrometer described has been used to make observations on all cloudless nights when the moon is low since December 1992, while the Fabry-Pérot interferometer has only been used routinely since July 1993 . The frequently poor observing conditions in Ireland, together with a site altitude of only $60 \mathrm{~m}$ above sea level, make it difficult to obtain long sequences of observations. Good-quality $\mathrm{OH}$ spectra were recorded, however, with an integration time of 5 min on 114 clear or partially clear nights during 1993 . The Fabry-Pérot interferometer is much more sensitive to scattered light and could not be operated nearly as frequently as the FTIR. Nevertheless, we believe that the results shown in this paper demonstrate the feasibility of obtaining meaningful geophysical measurements from Ireland in spite of the difficulties usually encountered.

\section{Acknowledgments}

We acknowledge the contributions of N. E. McKeith in initiating this program of research at Maynooth, and of 
Table 3 Internal gravity wave characteristics, 27-28 September 1993, Maynooth.

\begin{tabular}{lcc}
\hline Quantity & Symbol & Value \\
\hline Period & $\tau$ & $120 \mathrm{~min}$. \\
Angular frequency & $\omega$ & $8.7 \times 10^{-4}$ \\
Horizontal wave speed & $V_{h}$ & $155 \mathrm{~m} \mathrm{~s}^{-1}$ \\
Horizontal direction & $\theta$ & - \\
Horizontal wavelength & $\lambda_{h}$ & $1119 \mathrm{~km}^{-1}$ \\
Horizontal wavenumber & $k_{x}$ & $5.6 \times 10^{-6} \mathrm{~m}^{-1}$ \\
Vertical wavelength & $\lambda_{z}$ & $46 \mathrm{~km}^{-1}$ \\
Vertical wavenumber & $k_{z}$ & $1.38 \times 10^{-4} \mathrm{~m}^{-1}$ \\
Vertical wave speed & $V_{z}$ & $6.35 \mathrm{~m} \mathrm{~s}^{-1}$ \\
Zenith angle of propagation & $\psi$ & $2.35^{\circ}$ \\
\hline
\end{tabular}

Assuming a background wind of zero $\mathrm{m} \mathrm{s}^{-1}$.

D. Gleeson, M. Martin, W. Lanigan, and N. Murphy for fabricating much of the ancillary apparatus. The help of graduate students J. Keating, D. Doyle, A. Alcock, D. Horgan, J. Galligan, and E. Griffin in the development and testing of the instruments and observatory described is gratefully appreciated. This work was supported by EOLAS, the Irish Science and Technology agency, under grant numbers RE/ 176/89 and SC/91/141. R. J. Niciejewski acknowledges support from NSF under grant INT 9312085.

\section{References}

1. A. Krieger and F. Arnold, "Evidence of upper stratospheric aerosols from balloon-borne mass spectrometers," Geophys. Res. Lett. 19. 2301-2304 (1992).

2. K. Kita, N. Iwagami, and T. Ogawa, "Rocket observations of oxygen night airglows: excitation mechanisms and oxygen atom concentrations,"' Planet. Space Sci. 40, 1269-1288 (1992).

3. T. L. Killeen and R. G. Roble, "Thermospheric dynamics: contributions from the first 5 years of the Dynamics Explorer program," Rev. Geophys. 26, 329-367 (1988).

4. R. E. Bills, C. S. Gardner, and C.-Y. She, "Narrowband lidar technique for sodium temperature and Doppler wind observations of the upper atmosphere,"' Opt. Eng. 30(1), 13-21 (1991).

5. H. K. Myrab $\varnothing$ and C. S. Deehr, "Mid-winter hydroxyl night airglow emission intensities in the northern polar cap,' Planet. Space Sci. 32, 263-271 (1984).

6. J. H. Hecht, R. L. Walterscheid, G. G. Sivjee, A. B. Christensen, and J. B. Pranke, "Observations of wave-driven fluctuations of $\mathrm{OH}$ nightglow emission from Sondre Stromfjord, Greenland,' J. Geophys. Res. 92, 6091-6099 (1987)

7. R. A. Viereck and C. S. Deehr, "On the interaction between gravity waves and the $\mathrm{OH}$ Meinel (6-2) and the $\mathrm{O}_{2}$ atmospheric $(0-1)$ bands in the polar night airglow,' J. Geophys. Res. 94, 5397-5404 (1989).

8. Ground-based Optical Aeronomy science steering committee for Aeronomy Programme of National Science Foundation, "Overview," $C E$ DAR: Coupling, Energetics, and Dynamics of Atmospheric Regions, 1, 1-36 (1986).

9. G. E. Thomas, "Mesospheric clouds and the physics of the mesopause region,'” Rev. Geophys. 29, 553-575 (1991).

10. F. Yi, J. Klostermeyer, and R. Rüster, "VHF radar observations of gravity wave critical layers in the polar summer mesopause region,' Ann. Geophys. 10, 887-894 (1992).

11. V. Makhlouf, I. Eewan, J. Isler, and T.-F. Tuan, “On the importance of purely gravitationally induced density, pressure, and temperature variations in gravity waves," J. Geophys. Res. 95, 4103-4111 (1990).

12. M. J. Taylor and R. Edwards, "Observations of short period mesospheric waves patterns: in situ or tropospheric wave generation," Geophys. Res. Lett. 18, 1337-1340 (1991).

13. B. S. Dandekar and D. J. Davis, "Calibrations of the airglow photometers and spectrometers," Appl. Opt. 12, 825-831 (1983).

14. T. L. Killeen, B. C. Kennedy, P. B. Hays, D. A. Symanow, and D. H. Ceckowski, "Image plane detector for the Dynamics Explorer FabryPérot interferometer,' Appl. Opt. 22, 3503-3513 (1983).
15. J. W. Meriwether, Jr., P. Shih, T. L. Killeen, V. B. Wickwar, and R. G. Roble, "Nighttime thermospheric winds over Sondre Stomfjord,' Geophys. Res. Lett. 11, 931-934 (1984).

16. G. G. Sivjee, "Airglow hydroxyl emissions,"' Planet. Space Sci. 40, 235-242 (1992).

17. D. N. Turnbull and R. P. Lowe, "Vibrational population distribution in the hydroxyl night airglow,' Can. J. Phys. 61, 244-250 (1983).

18. F. H. Mies, "Calculated vibrational probabilities of $\mathrm{OH}\left(\mathrm{X}^{2} \Pi\right)$,' J. Mol. Spectrosc. 53, 150-188 (1974).

19. V. I. Krassovsky, N. N. Shefov, and V. I. Yarin, "Atlas of the airglow spectrum 3000-12400 A," Planet. Space Sci. 9, 883-915 (1962).

20. J. A. Coxon, "Optimum molecular constants and term values for the $\mathrm{X}^{2} \Pi(v \leq 5)$ and $\mathrm{A}^{2} \Sigma^{+}(v \leq 3)$ states of $\mathrm{OH}, '$ Can. J. Phys. 58, 933 949 (1980).

21. T. L. Killeen and P. B. Hays, "Doppler line profile analysis for a multichannel Fabry-Pérot interferometer,' Appl. Opt. 23, 612-620 (1984).

22. R. J. Niciejewski and J. H. Yee, "Airglow rotational temperature measurements during the ALOHA-90 campaign," Geophys. Res. Lett. 18, 1353-1356 (1991)

23. R. R. Garcia and S. Solomon, "The effect of breaking gravity waves on the dynamics and chemical composition of the mesosphere and lowe thermosphere,' J. Geophys. Res. 90, 3850-3868 (1985).

24. R. T. Clancy and D. W. Rusch, "Climatology and trends of mesospheric (58-90 km) temperatures based upon 1982-1986 SME limb scattering profiles,' J. Geophys. Res. 94, 3377-3393 (1989).

25. A. E. Hedin, "Extension of the MSIS thermospheric model into the middle and lower atmosphere," J. Geophys. Res. 96, 1159-1172 (1991).

26. J. F. Noxon, "Effects of internal gravity waves upon airglow temperatures," Geophys. Res. Lett. 5, 25-27 (1978).

27. D. J. Baker and A. T. Stair, Jr., "Rocket measurements of the altitude distributions of the hydroxyl airglow,'” Physica Scripta 37, 611-622 (1988).

28. R. G. H. Greer, D. P. Murtagh, I. C. McDade, P. H. G. Dickinson, L. Thomas, D. B. Jenkins, J. Stegman, E. J. Llewellyn, G. Witt, D. J. MacKinnon, and E. R. Williams, "Eton 1: a data base pertinent to the study of energy transfer in the oxygen nightglow,' Planet. Space Sci. 34, 771-788 (1986)

29. R. H. Wiens, S.-P. Zhang, R. N. Peterson, and G. G. Shepherd, "MORTI: A mesopause oxygen rotational temperature imager,' Planet. Space Sci. 39, 1363-1375 (1991).

30. D. W. Tarasick and G. G. Shepherd, "Effects of gravity waves on complex airglow chemistries 2. OH emission," J. Geophys. Res. 97, 3195-3208 (1992)

31. A. H. Manson, "Gravity wave horizontal and vertical wavelengths: an update of measurements in the mesopause region,"' J. Atmos. Sci. 47 2765-2773 (1990).

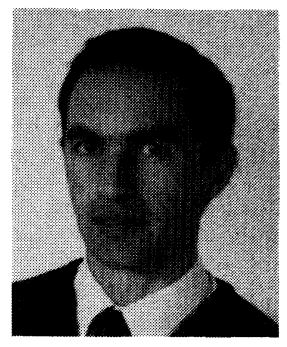

Francis J. Mulligan received BSc and $\mathrm{PhD}$ degrees in experimental physics from St. Patrick's College, Maynooth, in 1978 and 1981, respectively. He was a lecturer in physical sciences at the National Institute for Higher Education in Dublin from 1981 to 1984 . He became a member of the faculty at St. Patrick's College, Maynooth, in 1984, where he is currently a senior lecturer. Dr. Mulligan's main research interests are in the fields of atmospheric sensing and electron optics. He has spent periods of research at City College, New York, Brookhaven National Laboratory, and the University of Michigan. In recent years he has concentrated on instrumentation used for ground-based aeronomical studies.

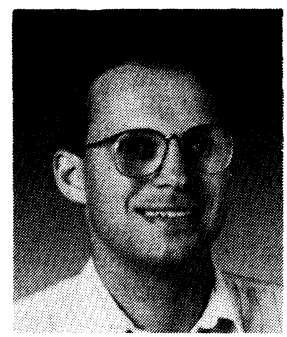

Rick J. Niciejewski received an MSc in 1981 and a PhD in 1987 from the University of Western Ontario in London, Canada. He joined the Space Physics Laboratory of the Atmospheric, Oceanic, and Space Science Department of the University of Michigan as a postdoctoral fellow in 1987, and became an assistant research scientist in 1990. His current research interests are in the field of experimental aeronomy including optical observations of the terrestrial airglow and aurora. Primary topics of interest are the thermodynamics and weather of the terrestrial upper atmosphere and the application of state-of-the-art technology to remote diagnostics. 